\title{
Arbor
}

\section{Monodromía y ecuaciones fuchsianas en la obra de H. Poincaré}

\author{
José Manuel Aroca Hernández-Ros
}

Arbor CLXXVIII, 704 (Agosto 2004), 625-643 pp.

Las cinco memorias de Poincaré sobre funciones y grupos fuchsianos publicadas en Acta Mathematica entre 1882 y 1884 constituyen la culminación de la teoría geométrica de funciones y el origen de un gran número de problemas de la Matemática actual. La cuarta memoria, dedicada a los grupos de monodromía de las ecuaciones diferenciales lineales de tipo Fuchs, es la menos conocida pese a contener resultados interesantes tanto en torno al problema de Riemann-Hilbert como a la estructura de los espacios de móduli de curvas algebraicas. En esta nota, expuesta en un ciclo de conferencias sobre Poincaré en su sesquicentenario, organizado en la Real Academia de Ciencias, se exponen las conexiones de esta memoria con resultados recientes de la teoría de Galois diferencial.

\section{Introducción y un poco de historia}

Las cinco memorias publicadas por Poincaré en Acta Mathematica entre 1882 y 1884, representan, en palabras de Darboux (Éloge historique d'Henri Poincaré [4]), la culminación de la parte más brillante de sus trabajos, y su mayor titulo de gloria. En 1880 Poincaré, entonces un joven de 26 años, presenta una memoria, que no fue publicada hasta 1923 , al concurso para el gran premio de Ciencias de la Academia convocado con un tema fijo: perfeccionar en algún punto importante la teoría de 


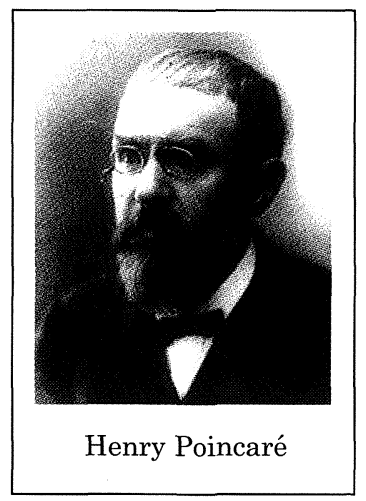
modo casi definitivo, tras escribir una de las piezas más brillantes de la Matemática. Las líneas de despedida escritas al final de su ultima memoria son muy características de su forma de ser:

Esto basta para hacer comprender que en las cinco Memorias de Acta Matemática que he consagrado al estudio de las transformaciones fuchsianas y kleinianas, no he hecho más que hacer aflorar un tema muy amplio que proporcionará sin duda a los geómetras la ocasión de hacer numerosos e importantes descubrimientos.

Pero de hecho estas memorias son mucho más, constituyen la culminación de la teoría geométrica de funciones, y son una perfecta síntesis de todos los conocimientos de análisis, álgebra, geometría y topología de su tiempo.

El enorme interés del trabajo de Poincaré sobre ecuaciones diferenciales lineales, la cantidad de matemáticas a que dicho trabajo ha dado lugar en los ciento veinte años que han trascurrido desde su publicación, y la polémica franco - alemana sobre la paternidad de las funciones fuchsianas - automorfas, son circunstancias que han hecho que proliferen los ensayos y estudios dedicados tanto a glosar este trabajo como a relatar su historia. Incluso el propio Poincaré dejo descrito con total precisión el momento en que se le ocurrió la idea central de usar la geometría hiperbólica.

Pero a pesar de la cantidad de literatura existente, hay una de las memorias de Acta, la cuarta, que ha quedado marginada y apenas se ha comentado, y eso que en ella se sientan precisamente las bases para alcanzar el objetivo final de su autor, resolver las ecuaciones diferenciales lineales con coeficientes algebraicos, y pese también a las conexiones de esta memoria con problemas tan conocidos como el problema de Rie- 
Monodromía y ecuaciones fuchsianas en la obra de...

mann-Hilbert o el de la estructura de los espacios de móduli de curvas de género dado.

John Stillwell, traductor al inglés de una selección de los artículos sobre funciones fuchsianas de Poincaré ([24]), se refiere a esta cuarta memoria en dos ocasiones en la Introducción del traductor (ver [24]), que precede a los textos seleccionados; en la primera de ellas dice:

Finalmente he introducido el cuarto artículo de Acta, porque es el más ambicioso de los suyos (de Poincaré) y continúa siendo relevante en las matemáticas de hoy en día.

En la segunda ocasión explica la razón por la cual, a su entender, esta cuarta memoria es la menos comentada.

Mientras gran parte de los tres primeros artículos de Acta ha sido asimilada y ha alcanzado los libros de texto, el cuarto continúa siendo misterioso y de difícil comprensión, en parte debido al retroceso de Poincaré, en los primeros parágrafos, a la teoría pregeométrica de ecuaciones diferenciales, pero también porque cuando finalmente reduce sus teoremas a topología, debe enfrentarse a variedades multidimensionales difíciles de manejar apropiadamente con los conocimientos de su época.

La opinión de Stillwell que aparece en esta ultima frase, fue también la opinión de Klein ([11]) que acusaba a Poincaré de que sus trabajos, publicados demasiado rápidamente, contenían mucho de provisional e incluso mucho de falso o exagerado y que eso resultaba muy patente, en particular, en esta cuarta memoria de Acta. Poincaré, siempre según Klein: tropezaba con dificultades, insuperables en ese momento, porque debía tratar con variedades abiertas a las cuales no se les podía asignar una frontera.

Sin embargo, en mi opinión, la memoria no es oscura, es por el contrario un buen exponente de la claridad de ideas de Poincaré y de su prodigiosa intuición geométrica. Poincaré vio perfectamente dónde estaban los puntos más espinosos, y la carencia de las técnicas matemáticas necesarias para sobrepasarlos no le impidió prever el camino por el cual hoy en día algunas de esas dificultades han sido superadas.

No pretendo hacer en estas líneas un trabajo de historiador, no es mi oficio y sería locura practicar el intrusismo en un terreno en el que ya existe una referencia excepcional, el magnifico libro de Jeremy Gray: $L i$ near differential equations and Group theory from Riemann to Poincaré [9]. Pero, ya que he citado algunas opiniones de Klein sobre el trabajo de 
Poincaré, no puedo resistir la tentación de dedicar unas líneas a su polémica (prácticamente unilateral) con éste, sobre el descubrimiento de las funciones fuchsianas - automorfas. Gray ([9]) que analiza exhaustivamente la polémica y la correspondencia entre ambos en el periodo 18801883 (pp. 295-310 op. cit.), afirma caritativamente:

No fue ni sencilla ni principalmente una carrera, la generosidad de cada uno de los hombres hacia el otro, hizo que fuera un autentico esfuerzo cooperativo.

Aunque luego matiza estas palabras añadiendo que en cualquier caso y dados su carácter y las circunstancias de la matemática alemana de su época, Klein se vio forzado a considerar que participaba en un auténtico reto. Eso parece bastante claro, en 1928 el propio Klein afirma en su Entwicklung der Mathematik im 19 Jahrhundert [12]

El precio que tuve que pagar por mi obra fue extraordinariamente alto; $m i$ salud se colapsó completamente........Mi actividad productiva real pereció en 1882....En consecuencia Poincaré tuvo el campo libre y, hasta 1884, publicó sus cinco grandes articulos sobre las nuevas funciones.

Estas frases no suenan precisamente a esfuerzo cooperativo. Pero hay mas: hace ya veinte años, el Seminario de historia de la Matemática del Instituto Henri Poincaré ([1], [16]), publicó una serie de cartas, recopiladas por P. Dugac, dirigidas a Poincaré por diversos matemáticos, cartas que no han sido recogidas por Gray, y que arrojan una luz especial sobre la polémica citada. En unas de ellas, escrita por Hermite [16] el 8 de Julio de 1881, aparece lo siguiente:

Lo que Vd. (Poincaré) me dice de que ha sido informado sobre la malquerencia de Mr. Klein, tanto contra Vd. como contra Mr. Fuchs y Mr. Picard me preocupa mucho.

A continuación Hermite trata de despejar las brumas que enturbian las relaciones científicas entre Poincaré y Klein:

He preguntado a Mr. Darboux, que lo conoce personalmente (a Klein), si sabía si Mr. Klein tiene respecto a los franceses la misma inquina que tantos de sus compatriotas. Debo decirle que, a mi entender, la respuesta de Mr. Darboux, si no destruye los informes que Vd. ha recibido, al menos los pone seriamente en duda. 
Monodromía y ecuaciones fuchsianas en la obra de...

Sigue explicandole que Darboux había enviado a Alemania a un joven egresado de la École Normal, Mr. Brunel, para seguir los cursos de Klein y que:

Si yo he comprendido bien, Mr. Darboux ha sabido por Mr. Brunel que Mr. Klein expone a sus alumnos los últimos trabajos que Vd. ha publicado en los Comptes Rendues, haciendo elogio de ellos.

Lamentablemente el mes anterior Brunel había escrito a Poincaré la primera de una serie de cartas de las que parece deducirse que Hermite no había comprendido bien a Darboux.

En primer lugar, Brunel en su primera carta deja patente que no era precisamente germanófilo:

Siguiendo la costumbre alemana, comencé por presentarme, pero no hay que creer que porque me haya adaptado a una costumbre de los alemanes, me encuentre bien en medio de este pueblo. Cuanto más lo conozco más lo detesto.

En el párrafo siguiente, Brunel echa por tierra los esfuerzos conciliadores de Darboux explicando la posición de Klein respecto a los trabajos de Poincaré.

Tomando un Compte Rendue al azar, nos dijo Klein, encuentro una nota sobre las funciones fuchsianas. El nombre de fuchsianas le permitió a Mr. Klein hacer algunas chanzas más o menos finas que cosquilleaban las orejas alemanas que las escuchaban.

Klein también nos ha dicho: Yo protesto contra el nombre de funciones fuchsianas. Es de Riemann de quien viene la idea fundamental, y es Schwarz quien tiene el mérito de haber aplicado la idea de Riemann. Más tarde, yo mismo he trabajado también en esa dirección. En cuanto a Mr. Fuchs que sólo se ha ocupado una vez de estas cuestiones, lo único que ha conseguido mostrarnos es una cosa: que no ha comprendido absolutamente nada.

Jean Dieudonné en una conferencia conmemorativa del centenario del comienzo de los trabajos de Poincaré sobre las funciones fuchsianas, pronunciada en el congreso del Grupo de Matemáticos de expresión latina de Luxemburgo [5], se hace eco de la indignación de Klein ante el nombre de funciones fuchsianas cuando dice, aludiendo precisamente a los trabajos de Riemann, Schwarz y el propio Klein:

Observando esta serie de trabajos, se concibe fácilmente el asombro un poco escandalizado de Klein cuando constata que, en las primeras notas de los Comptes Rendues, sólo se cita a Fuchs, y que se coloca bajo el patronazgo de 
éste la inmensa teoría que se edifica en ellos. Este asombro debió tornarse estupefacción cuando, respondiendo a Klein, Poincaré reconoce de buen grado que ignora todo sobre sus trabajos, que no ha leido nada de Riemann, al que sólo conoce de oídas, y que no sabe nada ni del género de una superficie, ni del principio de Dirichlet.

Una vez que Poincaré conoce los trabajos de Klein, en un alarde de generosidad incluso da su nombre a unos nuevos objetos, los grupo kleinianos, pero este hecho disgusta aún más a Klein, según Brunel, por hacerlo aparecer en segunda fila.

De nuevo, ahora a principios de julio, Brunel escribe a Poincaré, citando literalmente lo que le dice Klein en una entrevista:

Leyendo lo que Poincaré acaba de publicar en los Comptes Rendues, se podrá imaginar que le he escrito,..., le he escrito que todo lo que ha hecho es conocido y está probado desde hace mucho tiempo. Además repruebo a Mr. Poincaré el publicar demasiado deprisa.... Mr. Schotty ha publicado en el vol. 83 del Journal de Brochardt, una memoria que se ocupa del caso del polígono con borde no conexo; podría ser que Mr. Poincaré llamase ahora a otro tipo de funciones, funciones schottkianas; no hay razón para detenerse.

Pero Brunel no se limita al cotilleo, sus cartas contienen afirmaciones muy precisas en relación con lo que conocía Klein sobre funciones automorfas antes de leer los sucesivos artículos y las cartas de Poincaré, así como sobre la veracidad de sus afirmaciones de ir por delante de éste:

Yo le puedo afirmar que todavía no he encontrado ninguna traza, ni en las memorias de Klein, ni en su curso del Polytechnicum, de funciones análogas a una función zeta-fuchsiana ó theta-fuchsiana. Además Mr. Klein no ha abordado nunca estas cuestiones en todas las conversaciones que hemos tenido sobre Vd.

En una de mis entrevistas con él, Mr. Klein, me explicó que había sabido tras un encuentro con Mr. Weiestrass en pascua, que algunas de las ideas de Vd. existían antes, de forma algo distinta, en el cerebro del matemático berlinés. Pero esto no me parece gran cosa.

En cualquier caso, está fuera de toda duda que hay que atribuir a Poincaré la mayor parte de la teoría de funciones fuchsianas, especialmente su uso genial en dicha teoría de la geometría hiperbólica. Y también habría que atribuirle los resultados contenidos en la cuarta memoria de Acta, de la que hablaremos a continuación, y que reflejan la intuición, calificable de profética, del genial matemático francés. 
Monodromía y ecuaciones fuchsianas en la obra de...

\section{Las tres primeras memorias de acta mathematica}

Según reconoce el propio Poincaré, el origen de su trabajo está en un problema estudiado por Fuchs [8] que consiste en lo siguiente:

Dada una ecuación diferencial lineal de segundo orden con coeficientes funciones racionales de una variable compleja:

$$
y^{\prime \prime}+a_{1}(z) y^{\prime}+a_{2}(z) y=0
$$

y dadas dos soluciones $C$-linealmente independientes de la ecuación, $y_{1}$, $y_{2}$, averiguar bajo qué hipótesis complementaria sobre la ecuación diferencial, la ecuación funcional

$$
\text { (*) } \quad g(z)=\frac{y_{1}(z)}{y_{2}(z)}=u
$$

tiene solución $z=z(u)$ meromorfa en $u$.

Aunque Fuchs no explica el origen del problema, es claro que es una generalización, a una ecuación diferencial lineal general de segundo orden, del problema de inversión del cociente de periodos de una integral elíptica, (origen de la teoría de funciones modulares), y del problema análogo para la ecuación hipergeométrica, que se remonta a Jacobi, Gauss, y Legendre.

En su trabajo Fuchs supone que las singularidades de la ecuación diferencial son regulares y que las soluciones en ellas no tienen términos logarítmicos, a continuación prueba que si $g\left(z_{0}\right)=u_{0}$ para un punto singular $z_{0}$, la ecuación (*) se resuelve localmente en un entorno de $u_{0}$, y la solución es holomorfa en dicho entorno siempre y cuando los exponentes característicos en $z_{0}$ verifiquen unas condiciones complementarias (luego simplificadas por Poincaré). A continuación y de forma no suficientemente justificada, Fuchs admite que la existencia de solución local del problema de inversión lleva consigo la existencia de solución global.

En su memoria para la Academia, Poincaré caracteriza exactamente las condiciones sobre los exponentes característicos en las singularidades para que el problema de inversión local tenga solución y da un contraejemplo a la existencia de solución global del problema, en las condiciones propuestas por Fuchs. 
Pero esto es solo el principio de la obra de Poincaré. El planteamiento del problema de inversión cambia completamente tras los trabajos de Kummer, Dedekind, Riemann y Klein, que culminaron con la descripción de las funciones modulares, estableciendo que las funciones $g(z)$ de $\left(^{*}\right)$ correspondientes a la ecuación hipergeométrica, representan conformemente el semiplano superior sobre un triángulo cuyos lados son segmentos o arcos de circunferencia, de modo que las representaciones correspondientes a dos determinaciones difieren en la acción de un elemento de $G S L_{2}(R)$. Como observa Dieudonné [5]:

Ya no se trata de estudiar el cociente de dos integrales de una ecuación diferencial, sino de determinar, para un subgrupo de $G$, un dominio fundamental simple $F$, tal que toda orbita de $G$ en el semiplano superior corte a $F$ en un único punto.

Las funciones a considerar son entonces las funciones automorfas, es decir invariantes, por la acción de subgrupos del grupo G. Se ha escrito mucho, incluso lo ha hecho el propio Poincaré, sobre la utilización de la geometría hiperbólica en este campo, en el libro de Gray [9] por ejemplo se puede encontrar una buena y detallada descripción elemental del trabajo de Poincaré. Señalemos aquí únicamente que la primera memoria de Acta esta dedicada a los grupos fuchsianos, que se introducen como grupos de movimientos para la geometría hiperbólica que preservan una teselación del semiplano superior, o lo que es lo mismo como grupos de transformaciones de Möbius con coeficientes reales que actúan de modo discontinuo en dicho semiplano. Estas condiciones llevan consigo la existencia de funciones analíticas no constantes que son automorfas para los grupos fuchsianos, estas son las funciones fuchsianas. La segunda memoria de Acta está dedicada al estudio de estas funciones.

Para construir las funciones fuchsianas correspondientes a un grupo $\Gamma$, Poincaré traslada su geometría (eso lo hace al final del primer articulo) al círculo unidad mediante la transformación habitual:

$$
\delta(z)=\frac{z+i}{z-i}
$$

y comienza con la descripción de las funciones theta - fuchsianas, por medio de series indexadas por $\Gamma$ :

$$
\Theta_{R, n}=\sum_{\gamma \in \Gamma} R(\gamma)\left(\frac{d \gamma}{d z}\right)^{n}
$$


Monodromía y ecuaciones fuchsianas en la obra de...

donde $R$ es una función racional y $n$ un número natural. Estas series, para $n \geq 2$, son absolutamente convergentes en el disco unidad salvo en los polos de $R$ y verifican que:

$$
\gamma \in \Gamma, \quad \gamma(\mathrm{z})=\frac{\mathrm{az}+\mathrm{b}}{\mathrm{cz}+\mathrm{d}} \Rightarrow \Theta_{R, n}(\gamma)=(c z+d)^{2 n} \Theta_{R, n}(z)
$$

Entonces los cocientes de las funciones definidas por dos de estas series correspondientes al mismo entero $n \geq 2$, son funciones meromorfas en el disco unidad y son exactamente las funciones automorfas para el grupo $\Gamma$, es decir son las funciones fuchsianas. Poincaré estudia los ceros y polos de estas funciones, además prueba que todas las funciones fuchsianas correspondientes a un mismo grupo $\Gamma$ generan un cuerpo que es un cuerpo de funciones racionales sobre una curva algebraica cuyo género coincide con el de $\Gamma$, es decir con el de la superficie cociente del disco por la acción de este grupo. Por último caracteriza las ecuaciones lineales de segundo orden resolubles mediante funciones fuchsianas.

La tercera memoria de la serie está dedicada a los grupos kleinianos. Los grupos kleinianos son grupos de transformaciones de Möbius que actúan de modo discontinuo en el plano y no son fuchsianos, la existencia de este tipo de grupos fue señalada a Poincaré por Klein, de ahí su nombre, que como hemos señalado no gustaba mucho a Klein.

Poincaré demuestra en primer lugar que toda transformación de Möbius es producto de un número par de inversiones, de hecho va mas lejos y prueba que el grupo de proyectividades continuas de la recta proyectiva compleja está generado por las inversiones. A continuación observa que toda circunferencia del plano real es el ecuador de una esfera del espacio y que la inversión respecto a dicha esfera deja invariante el plano e induce en él la inversión respecto a la circunferencia de partida. Entonces una transformación de Möbius del plano es la sombra, en expresión de Stillwell, de una transformación del espacio producto de un número par de inversiones respecto a esferas, o sea, cuando las esferas se eligen adecuadamente, de un movimiento del espacio hiperbólico tridimensional. De nuevo Poincaré recurre a la geometría hiperbólica, esta vez en un espacio de dimensión tres, para el estudio de los grupos discontinuos de transformaciones de Möbius.

Los conjuntos límite de los grupos fuchsianos están contenidos en el borde del disco unidad, en cambio los de los grupos kleinianos son curvas en general no analíticas. De hecho Poincaré conjeturó que son curvas no 
634

José Manuel Aroca Hernández-Ros

diferenciables en un conjunto denso de puntos, conjetura que fue probada por Fricke y Klein [7]en 1897, aunque la naturaleza fractal de la curva límite solo ha sido reconocida muy posteriormente por Mandelbrot [18].

\section{Cálculo del grupo de monodromía de una ecuación}

La cuarta memoria de Acta, esta destinada a la generalización del problema de Fuchs a las ecuaciones diferenciales lineales de orden arbitrario; en este caso hay mas de dos soluciones independientes, por lo que no tiene sentido estudiar el grupo cuya acción regula las distintas determinaciones del cociente de dos de ellas, y parece mas natural preguntarse por el grupo que deja invariante el espacio de soluciones.

Si pensamos en ecuaciones algebraicas, en lugar de en ecuaciones diferenciales, las transformaciones naturales para las ecuaciones polinómicas son aquellas que conservan sumas y productos, es decir los automorfismos de cuerpos, y si consideramos la extensión $\Sigma$ del cuerpo $K$ donde están los coeficientes de una ecuación $f(x)=0$, obtenida al adjuntar a $K$ las raíces de la ecuación, el grupo de Galois de $\Sigma$ respecto a $K$, es decir el grupo de automorfismos de $\Sigma$ que dejan invariantes los elementos de $K$, es precisamente el análogo algebraico del grupo que buscaba Poincaré. Cuando el cuerpo $K$ es el cuerpo $C(z)$ de funciones racionales en la variable $z$ con coeficientes complejos, la analogía es mas clara porque ese grupo es exactamente el grupo que traduce las distintas determinaciones de las funciones algebraicas definidas por la ecuación en dos variables, la $x$ explícita y la $z$ en los coeficientes, $f(x)=0$. Pero Poincaré no alcanza a ver esta analogía, que será descubierta por Picard unos años mas tarde. Pese a ello su construcción sobrepasa la monodromía y se aproxima indirectamente al grupo de Picard-Vessiot.

Poincaré considera una ecuación lineal general:

$$
F(y)=y^{(n)}+a_{1} y^{(n-1)}+\cdots+\alpha_{v} I=0
$$

con coeficientes funciones meromorfas sobre una superficie de Riemann compacta $\Sigma$, es decir: $\operatorname{los} a_{i}=a_{i}(z, x)$ son funciones racionales de dos variables complejas ligadas por una relación polinómica $\psi(z, x)=0$.

En estas condiciones, un punto de $\Sigma$ se dice singular de la ecuación si y sólo si es un polo de uno de sus coeficientes; el conjunto de puntos singulares de la ecuación $\operatorname{Sing}(F)$ es finito y para cada punto no singular, 
Monodromía y ecuaciones fuchsianas en la obra de...

existe un entorno abierto simplemente conexo en el cual los coeficientes son analíticos. En consecuencia la ecuación admite en dicho entorno un sistema local fundamental de soluciones, es decir un conjunto de $n$ soluciones $C$-linealmente independientes que generan el $C$-espacio vectorial de soluciones en el entorno en cuestión, $\left(y_{1}, y_{2}, \cdots, y_{n}\right)$.

Fijemos un punto singular $z$ y tomemos un lazo en $\Sigma \backslash \operatorname{Sing}(F)$ basado en $z, \gamma$. Por prolongación analítica del sistema fundamental $\left(y_{1}, y_{2}, \cdots\right.$, $y_{n}$ ) a lo largo de $\gamma$, llegamos a otro sistema fundamental $\left(u_{1}, u_{2}, \cdots, u_{n}\right)$, que está ligado necesariamente al primero por una relación:

$$
\left(\begin{array}{c}
u_{1} \\
\vdots \\
u_{n}
\end{array}\right)=M_{\gamma}\left(\begin{array}{c}
y_{1} \\
\vdots \\
y_{n}
\end{array}\right) ; \quad M_{\gamma} \in G L_{n}(C) .
$$

Siempre que nos mantengamos en $\Sigma \backslash \operatorname{Sing}(F), M_{\gamma}$ no cambia por deformaciones continuas de $\mathrm{g}$ y tenemos una representación lineal del grupo fundamental ó grupo de Poincaré $\Pi_{1}(\Sigma \backslash \operatorname{Sing}(F))$ :

$$
\begin{aligned}
\Pi_{1}(\Sigma \backslash \operatorname{Sing}(F)): & \rightarrow G L_{n}(C) \\
\gamma & \rightarrow M_{\gamma}
\end{aligned}
$$

Esta representación depende del sistema fundamental de soluciones elegido inicialmente, pero depende solamente módulo conjugación. Así queda definida una clase de conjugación de subgrupos de $G L_{n}(C)$, uno cualquiera de cuyos representantes se llama grupo de monodromía de la ecuación diferencial.

Poincaré aborda tres problemas, enunciados con toda precisión en la memoria. El primero es:

Hallar el grupo de una ecuación dada, tanto desde el punto de vista de cálculos numéricos, como desde el de la teoría de funciones.

Es decir se trata no solo de dar un método de calculo del grupo de monodromía de una ecuación concreta, sino de describir los elementos del grupo como función de los coeficientes de la ecuación y de la clase de homotopía del camino correspondiente.

Para ello se limita previamente, sin perdida de generalidad, al caso en que los coeficientes de la ecuación son funciones meromorfas en la esfera de Riemann y holomorfas en el infinito. En estas condiciones y tras un cambio de variable bien conocido (transformación de Tschirnhausen) la ecuación se escribe como: 


$$
y^{(n)}=\sum_{k=0}^{n-2}\left(\sum_{i=1}^{r} \sum_{h} \frac{A_{h k i}}{\left(x-\alpha_{i}\right)^{h}}\right) y^{(k)}
$$

entonces, supuesto que se fijan los puntos singulares, los elementos de las matrices de monodromía son funciones meromorfas de los $A_{h k i}$.

Poincaré prueba este resultado partiendo de un punto ordinario de la ecuación, $z_{0}$, y de un sistema fundamental local de soluciones en ese punto con matriz wronskiana en él igual a la identidad. Las soluciones del sistema local admiten un desarrollo de Taylor en potencias de $z-z_{0}$ con coeficientes polinómicos en los parámetros $A_{h k i}$, la convergencia en los parámetros se obtiene utilizando un teorema de Kowalewsky para ecuaciones en derivadas parciales. Se considera ahora un camino $\gamma$ en $\Sigma \backslash$ $\operatorname{Sing}(F)$ con origen en $z_{0}$ y por prolongación analítica se extiende el sistema local a lo largo de $\gamma$, los nuevos sistemas están relacionados con el inicial por una matriz cuyas entradas son también funciones enteras de los parámetros, así cuando se cierra el lazo se obtiene el resultado.

Hamburguer [15] había desarrollado previamente un algoritmo de calculo de la monodromía pero fue incapaz de comprobar la convergencia. Señalemos por último que casi veinte años mas tarde Picard [19] da una prueba directa de la convergencia del método de Poincaré que no precisa del teorema de Kowalewsky.

Una vez obtenido el resultado, las soluciones del sistema fundamental se pueden desarrollar en serie de potencias en los parámetros, y al considerar los coeficientes como funciones racionales de los polos se comprueba, por el mismo proceso anterior, que se pueden calcular por cuadraturas obteniéndose una serie en polilogaritmos.

Aquí llamamos polilogaritmos a una familia de funciones definidas por recurrencia de la forma siguiente:

Sea un conjunto finito de puntos de la esfera de Riemann $P_{C}^{1}$, y sea $U$ el recubridor universal de $P_{C}^{1} \backslash \Sigma$, con punto base 0 , si $\sigma$ es un subconjunto de $\sum$, llamamos $L_{\sigma}(u)$ a la función definida sobre $U$ por:

- $L_{\sigma}(u)=1$ si $\sigma=\varnothing$

- Si $\sigma=\left(\sigma_{1}, \sigma_{2}, \cdots, \sigma_{r}\right)$ y llamamos $\sigma^{\prime}=\left(\sigma_{1}, \sigma_{2}, \cdots, \sigma_{r-1}\right)$, entonces:

$$
L_{\sigma}(u)=\int_{0}^{u} \frac{L_{\sigma^{\prime}}(z) d z}{z-\sigma_{r}}
$$


Monodromía y ecuaciones fuchsianas en la obra de...

En este momento resulta muy interesante comparar los cálculos de Poincaré con los de Lappo Danielvskii en su solución al problema de Riemann - Hilbert, tal como los describe Beauville en su exposición para el Seminario Bourbakí [2] (no he tenido ocasión de consultar la obra original). Beauville escribe:

En 1928, el joven matemático ruso Lappo Danielvskï propone una aproximación totalmente diferente; escribe explícitamente las soluciones del sistema bajo la forma de series de polilogaritmos. El método es tan elegante que no me resisto a exponerlo aquí.

El método es exactamente el que Poincaré describe en 1880 , el único cambio es que la notación $\Lambda\left(x, \alpha_{1}, \alpha_{2}, \cdots, \alpha_{r}\right)$ que Poincaré usa para el polilogaritmo se cambia por $L_{\sigma}(u)$, y sin embargo curiosamente Beauville no lo cita (cómo ya he indicado no se si lo hace Danielvskii)

El lector no habituado a la forma de escribir de la época, puede quedar sorprendido por alguna de las afirmaciones de Poincaré, por ejemplo cuando afirma que los valores propios de una matriz son un sistema completo de invariantes de ésta, la afirmación es obviamente falsa a menos que pensemos que genéricamente todas las matrices son diagonalizables y por tanto, genéricamente, es decir para un abierto denso de matrices, la clase de conjugación de una matriz esta determinada por sus valores propios.

\section{Poincaré y el problema de Riemann-Hilbert}

En la sección cuarta de la memoria, y sin destacarlo especialmente Poincaré se plantea el problema de inversión para ecuaciones de orden $n$, es decir intenta averiguar si dado un grupo de matrices, existe una ecuación diferencial lineal que lo tenga como grupo de monodromía.

Hay dos líneas de trabajo muy importante en la matemática actual en las que se toca este problema. En la primera se substituye el grupo de monodromía, demasiado pequeño para reflejar suficientes propiedades de la ecuación, por un grupo mas amplio, el grupo de Galois diferencial, definido por Picard y Vessiot. Si partimos de una ecuación diferencial lineal con coeficientes en un cuerpo $K$ de funciones meromorfas sobre una superficie de Riemann, se puede construir una extensión $\Sigma$ de este cuerpo generada por un sistema fundamental de soluciones de la ecuación, $\Sigma$ con una derivada que extiende la de $K$ y tal que las constantes para esa derivada son exactamente los complejos. El grupo $\operatorname{Gal}_{\Delta}(\Sigma, K)$ de automor- 
fismos de $\Sigma$ que conmutan con la derivada y dejan invariantes los elementos de $K$ se llama grupo de Galois diferencial de la extensión. Como un automorfismo que conmuta con la derivada y deja invariantes los coe ficientes de la ecuación, transforma soluciones en soluciones, el grupo de Galois diferencial admite una representación lineal sobre el $C$ - espacio vectorial de soluciones de la ecuación. Es decir, asocia un homorfismo $G a l_{\Delta}(\Sigma, K) \rightarrow G L_{n}(C)$.La imagen de esta representación:

- Es un grupo algebraico de matrices

- Contiene al grupo de monodromía

- Si la ecuación es fuchsiana es exactamente el cierre del grupo de monodromía en la topología de Zariski $G L_{n}(C)$.

El problema inverso de la teoría de Galois diferencial, consiste en caracterizar los grupos lineales algebraicos que son grupos de una ecuación diferencial lineal. Al contrario que el correspondiente problema algebraico, este ha sido resuelto por J.P. Ramis, C. Mitschi y M.F. Singer, en sus distintas versiones locales y globales. El libro de Van der Put y Singer [26] es probablemente la mejor referencia para este problema. Señalemos únicamente, que dada la forma en que Poincaré aborda el problema con la descripción explícita de las formulas de inversión, sus resultados son cerrados para la topología de Zariski, por lo cual, cuando trabaja con ecuaciones fuchsianas, esta trabajando de hecho sobre el grupo de Galois diferencial.

Antes de hablar de la segunda línea de trabajo actual conectada con este problema, el teorema de Riemann-Hilbert, veamos brevemente que es lo que hace Poincaré.

Poincaré intenta construir un sistema completo de invariantes del grupo de monodromía de una ecuación y probar que los coeficientes de la ecuación son función de los invariantes del grupo. Su método de solución es indirecto basado en un cálculo de dimensiones, que hace contando el numero de parámetros de que dependen los coeficientes de una ecuación con singularidades prefijadas y el número de invariantes de los generadores del grupo de monodromía correspondiente. Como los invariantes son función de los coeficientes, si el número de parámetros de que dependen estos fuera mayor que el de invariantes se podrían invertir en general las fórmulas. Lamentablemente el número de parámetros de que efectivamente depende la ecuación es menor que la dimensión del grupo de monodromía. En consecuencia las formulas no se pueden invertir salvo para orden 2 , caso en que ambos números coinciden. Ahora bien, es fácil ver que hay singularidades de la ecuación, las singularidades aparen- 
Monodromía y ecuaciones fuchsianas en la obra de...

tes, que no generan monodromía. Entonces añadiendo un número suficientemente grande de singularidades aparentes, dice Poincaré, el numero de parámetros de la ecuación supera la dimensión del grupo. Entonces las formulas se pueden invertir siempre que se admita la presencia de suficientes singularidades aparentes.

Los problemas que obvia Poincaré son los siguientes:

- Necesita una clasificación módulo cambio analítico de variables de las ecuaciones diferenciales lineales, que le permita describir las componentes del espacio de móduli, y calcular efectivamente sus dimensiones.

- No puede limitarse a considerar el caso en que cada matriz de monodromía es diagonalizable, que al fin y al cabo es genérico, sino que necesita que todas estas matrices sean simultáneamente diagonalizables y esta condición ya no es genérica. Por tanto el calculo de la dimensión del grupo de monodromía no está suficientemente justificado.

- La coincidencia de las dimensiones del espacio de ecuaciones con singularidades prefijadas y el grupo de monodromía, no garantiza que las formulas de la aplicación que asocia a cada ecuación su grupo sean inversibles.

De todos modos merecería la pena desarrollar de modo formal y sin lagunas el programa que traza Poincaré en esta sección, sobre todo por su conexión con el llamado problema de Riemann-Hilbert.

Para explicar brevemente en que consiste este problema y su conexión con el trabajo de Poincaré, debemos volver a la ecuación diferencial lineal de orden $n, F(y)=0$ de tipo Fuchs, tomar el conjunto Sing $(F)=\left\{z_{1}\right.$, $\left.z_{2}, \cdots, z_{\mathrm{n}}\right\}$ de sus singularidades, y construir el grupo de Poincaré, $\Pi_{1}\left(P_{C}^{1}\right.$ $\backslash$ Sing $(F)$ ). Este grupo admite como generadores lazos basados en que encierran cada uno una sola singularidad y son tales que

$$
\gamma_{n} \gamma_{n-1} \cdots \gamma_{1}=1
$$

Las matrices de monodromía correspondientes a estos lazos verifican que

$$
M_{n} M_{n-1} \cdots M_{1}=I
$$

Entonces el problema de Riemann - Hilbert consiste en averiguar si todo sistema de matrices $\left\{M_{1}, M_{2}, \cdots, M_{r}\right\}$ con producto identidad se puede obtener de esta forma, es decir las son las matrices de los generadores del grupo de monodromía de una ecuación diferencial lineal. 
La historia de este problema está bien recogida el texto de Hille [17] o en el artículo ya citado de Beauville [2], por lo cual no la detallaremos aquí. Digamos únicamente, que si se pueden efectuar correctamente los cálculos de Poincaré, no solo se resuelve el problema inverso de la teoría de Galois diferencial para ecuaciones fuchsianas, es claro que se resuelve también el problema de Riemann-Hilbert. De hecho la solución de Lappo Danielvskii de este problema utiliza amplios fragmentos del trabajo de Poincaré como ya hemos indicado.

Mas aún Treibich [25] corrige un error presente en los trabajos de Plejmelj [20] y Birkhoff [3] sobre el problema de Riemann - Hilbert. Ese error es el de admitir la diagonalización simultanea como una propiedad genérica, que es casualmente el mismo que muchos años antes había cometido Poincaré. Estas dos observaciones son muestra suficiente de la influencia, no reconocida, de la obra de Poincaré en el trabajo de los primeros a que se atribuyen soluciones parciales del problema de Riemann - Hilbert.

\section{Existencia de ecuaciones de tipo dado y el problema de la uniformización}

Cómo el problema de encontrar ecuaciones con singularidades y monodromía prefijada, y sin singularidades aparentes solo se puede resolver, según Poincaré, en dimensión dos, y además Poincaré quiere encontrar ecuaciones con monodromía prefijada y singularidades regulares, no le queda mas remedio que precisar su clasificación de las ecuaciones lineales y demostrar que en toda clase hay una ecuación de tipo Fuchs. El resto de la cuarta memoria de Acta está dedicado a este objetivo.

Poincaré se limita, como ya hemos señalado, a ecuaciones de segundo orden sobre una superficie de Riemann compacta, a las que supone sin término en $z$ porque aplica la transformación de Tschirnhäusen, esto es a:

$$
\text { [1] }\left\{\begin{array}{lcc}
\frac{\mathrm{d}^{2} z}{\mathrm{dx}^{2}} & = & z \phi(x, y) \\
\psi(x, y) & = & 0
\end{array}\right.
$$

donde $\phi(x, y)$ es una función racional y $\psi(x, y)$ un polinomio. Dos de estas ecuaciones son equivalentes, es decir pertenecen al mismo tipo, si difieren en una transformación birracional de la superficie de Riemann. Como hay un modelo birracional no singular, módulo un cambio de va- 
Monodromía y ecuaciones fuchsianas en la obra de...

riable birracional, se puede suponer que localmente en cada punto de la curva $y$ es función holomorfa de $x$. Ahora dos ecuaciones que se escriben:

$$
\frac{d^{2} z}{d x_{1}^{2}}=z \phi\left(x_{1}\right), \quad \frac{d^{2} z}{d x_{2}^{2}}=z \phi\left(x_{2}\right)
$$

serán equivalentes cuando difieran en una transformación de Möbius general:

$$
x_{2}=\frac{a x_{1}+b}{c x_{1}+d}, \quad a d-b c=1
$$

Una vez escrita en esta forma la ecuación no tiene más singularidades que las estrictas, en cada singularidad la ecuación tiene una ecuación determinantal asociada de segundo grado, las raíces de esa ecuación se llaman exponentes característicos de la singularidad, y son invariantes del tipo de la ecuación.

Poincaré llama fuchsianas a las ecuaciones de segundo orden en las que, por inversión, $x$ es una función fuchsiana del cociente de las dos soluciones. Y llama tipo fuchsiano a una clase birracional de ecuaciones tales que para uno cualquiera de sus representantes, la diferencia entre los exponentes característicos sea de la forma $1 / n$ con $n$ entero, y que o bien el género $q$ de la superficie de Riemann se mayor o igual que dos o que sea menor que dos y:

$$
\sum_{1 \leq i \leq p} \alpha_{i}<2 q+p-2
$$

donde $p$ es el número de singularidades de la ecuación, y $\alpha_{i}$ es el valor absoluto de la diferencia entre los exponentes característicos de la singularidad i-ésima.

La prueba de que cada tipo fuchsiano contiene una ecuación fuchsiana, no está completa y este resultado que lleva consigo la uniformización de curvas de genero mayor o igual que dos por funciones automorfas fue considerado conjetura hasta que en 1907 el propio Poincaré [22] y Koebe [13] dieron independientemente la prueba definitiva. 


\section{Referencias}

[1] Brunel, G. (1986) Lettres de Georges Brunel a H. Poincare. En: Cahiers du Seminaire d'Histoire des Mathematiques. Ed: Inst. Henri Poincare. Paris. Vol. 7.

[2] Beauville, A. (1993) Monodromie des systèmes différentiels lnéaires à pôles simples sur la sphere de Riemann. En: Sem. Bourbaki 1992-93. Ed: Seminario Bourbaki. Paris. Vol. 765.

[3] Birkhoff, G. (1913) The generalized Hilbert problem for linear differential equations and the allied problems for linear difference and q-difference equations. Proc. Amer. Acad. 49.

[4] Darboux, G. (1995) Éloge historique d'Henri Poincare. En: OEuvres de Henri Poincaré. Ed: Jacques Gabay. Paris. Tome II.

[5] Dieudonne, J (1982) La découverte des fonctions fuchsiennes. En: Actes $d u$ VI congrès $d u$ regroupement des mathematiciens d'expression latine. $\mathrm{Ed}$ : Gauthier- Vilars. Paris.

[6] Favard, J. (1962) Cours d'analyse de l'École Polytechnique Tome III. Ed: Gauthier-Villars. Paris.

[7] Fricke, R. y Klein, F. (1897) Vorlesungen über die Theorie der automorphen Funktionen. Vol. I. Ed: Teubner, Leipzig.

[8] Fuchs, L. (1980) Über eine Klasse von Funktionen mehrerer Variabeln, durch Umkherung der Integrale von Lösungen der linearen Differentialgleichungen mit rationalen Coefficienten entstehen. Journ. für die reine und angew. Math. 89.

[9] Gray, J. (1986) Linear differential equations and group theory from Riemann to Poincaré. Ed: Birkhäuser, Boston.

[10] Katz, N. (1976) An overview of Deligne's work on Hilbert's twentyfirst problem. En: Mathematical developments arising from Hilbert's problem. En: A.M.S., Rhode Island. Vol. 2.

[11] Klein, F. (1928) Gesammelte mathematische Abhandlungen. Ed: Springer, Berlin.

[12] Klein, F. (1928) Entwicklung der Mathematik im 19 Jahrhundert. Ed: Springer, Berlin.

[13] Koebe, P. (1907) Zur Uniformizierung der beliebiger anlytische Kurven. Göttingen Nachrichten, pp. 191 - 210.

[14] Kuga, M. (1993) Galois dream. Ed: Birkhäuser, Boston.

[15] Hamburguer (1877) Bemerkungen über die Form der Integrale der linearen Differentialgleichungen mit veränderlichen Coefficienten. Journ. für die reine und angew. Math. 83.

[16] Hermite, Ch: Lettres de Ch. Hermite a H. Poincare. En: Cahiers du Seminaire d'Histoire des Mathematiques 7 Ed: Inst. Henri Poincare, Paris. Vol. 7.

[17] Hille, E. (1997) Ordinary differential equations in the complex domain. Ed: Dover, New York. 


\section{Monodromía y ecuaciones fuchsianas en la obra de...}

[18] Mandelbrot, B. (1983) Self inverse fractals osculated by sigma discs and the limit sets of inversion groups. Math Intelligencer $\mathbf{5}$.

[19] Picard, E (1983, tercera edición) Traité d'Analyse, Tome III. Ed: Gauthier-Villars, Paris.

[20] Plejmelj, J. (1908) Riemansnsche Funktionenscharen mit gegebene Monodromiegruppe. Mon. für Math. und Physik 19.

[21] Poincare, H. (1995) OEuvres, Tome II. Ed: Jacques Gabay Paris.

[22] Poincare, H. (1907) Sur l'uniformisation des functions analytiques. Acta Math. 31, pp. 1- 63.

[23] Rodriguez-Salinas, B., De Maria, L. (1998) Henri Poincaré. En: Historia de la matematica en el siglo XIX. Ed: Real Acad. Ciencias Madrid.

[24] Stilwell, J. (1985) Translator's introduction in H. Poincare: Papers on fuchsian functions. Ed: Springer, New York.

[25] Treibich Kohn, A. (1981) Un resultat de Plejmelj. En: Sem. E.N.S. 1978-79, Progress in Mathh. Ed: Birkhauser, Berlin. Vol. 37.

[26] Van der Put, M. Singer, M.: Galois theory of linear differential equations. Springer Berlin 2003.

[27] Yoshida, M. (1987) Fuchsian differential equations. Aspekte der Math. Ed: Vieweg and Sohn Braunschweig. Vol. 11 de Aspekte der Math.

\section{Notas}

Notas sobre la memoria de H. Poincaré «Sur les groupes des equations lineaires» terminada de escribir el 20 de octubre de 1883 y publicada en el tomo 4 de Acta Matemática (pp. 201-311) el 9 de febrero de 1884. 